\title{
Treatment for Lumbar Disc Herniation With Spinal Stenosisto Using Unilateral Biportal Endoscopy Technique: a Retrospective Study
}

\section{Baojie Shen}

The First People's Hospital of yuhang District https://orcid.org/0000-0003-4604-2583

\section{Xuwei Pan}

The First people's Hospital of Yuhang District

\section{Di Ruan}

The First People's Hospital of Yuhang

\section{Danfeng Dai}

The First People's Hospital of Yuhang

\section{Xiaoliang Qian}

The First People's Hospital of Yuhang

\section{Miaolin Zhang}

The First People's Hospital of Yuhang

\section{Yipeng Zhu}

The First People's Hospital of Yuhang

Zhichao Gao ( $\nabla$ leadyourdream@163.com )

The First People's Hospital of Yuhang

\section{Research article}

Keywords: Unilateral biportal endoscopy (UBE), lumbar disc herniation, spinal stenosisto, PEID group

Posted Date: January 11th, 2021

DOI: https://doi.org/10.21203/rs.3.rs-142781/v1

License: (c) (1) This work is licensed under a Creative Commons Attribution 4.0 International License. Read Full License 


\section{Treatment for lumbar disc herniation with spinal stenosisto using unilateral biportal endoscopy technique:a retrospective study}

Baojie Shen ${ }^{1}$, Xuwei Pan $^{1}$, Di Ruan ${ }^{1}$, Danfeng Dai ${ }^{1}$, Xiaoliang Qian ${ }^{1}$, Miaolin Zhang ${ }^{1}$, Yipeng Zhu ${ }^{1}$ and Zhichao Gao* ${ }^{1}$

Department of Orthopaedics, The First People's Hospital of Yuhang District, Hangzhou, China,369 Yingbin Road, Hangzhou 313000, China

Baojie Shen: 2596937672@qq.com; Xuwei Pan: 294757657@qq.com;

Di Ruan: 625829296@qq.com; Danfeng Dai: 342912257@qq.com

Xiaoliang Qian: 562152099@qq.com; Miaolin Zhang: zhhzju@sina.com

Yipeng Zhu: 545610658@qq.com; Zhichao Gao: leadyourdream@163.com

\section{ABSTRACT}

Background: This paper is to describe Unilateral biportal endoscopy (UBE) in the treatment of lumbar disc herniation with spinal stenosis and to investigate the efficacy and safety of unilateral biportal endoscopy in the treatment of this kind of lumbar disc herniation with spinal stenosis in elderly patients.

Method: Retrospective analysis of clinical and radiological data of patients receiving UBE or PEID treatment at the First People's Hospital of Yuhang District, Hangzhou, China, from July 2018 to June 2020 was performed.

Result: The operation time of the UBE group was better than that of the PEID group $(\mathrm{P}<0.05)$. Based on the comparative analysis between the two groups, both the ODI score and the pain index were not statistically significant $(\mathrm{P}>0.05)$ but the intra-group comparison before and after the treatment was statistically significant $(\mathrm{P}<0.05)$. No marked intraoperative and postoperative complications occurred in the UBE group. In the PEID group, one patient developed transient pain in the lower extremities 3 days after surgery, while another patient developed numbness and discomfort in the lower extremities. Both groups were treated conservatively and recovered. During the follow-up, there were no serious adverse events that required another operation.

Conclusion: UBE technology can achieve the same clinical effects as percutaneous 
endoscopic technology in the treatment of LDH with spinal stenosis. UBE technology is easy to apply during operation. For surgical instruments, UBE can not only use special instruments for endoscopes but also relaxes the conditions for application and use of open surgical instruments. During the operation, the operation is more effective and safe, and the learning curve is milder than that of the percutaneous endoscopic technique, which can be used as one of the options for surgical treatment of LDH and spinal stenosis in the future.

\section{Introduction}

Lumbar disc herniation (LDH) clinically manifests with waist and leg pain due to the compression and stimulation of the surrounding tissues or nerve roots due to the lumbar disc degeneration, backward protrusion of nucleus pulposus or the emergence of nucleus pulposus after the rupture of intervertebral disc annulus fibrosus [1]. Meanwhile, due to the posterior protrusion of the nucleus pulposus, thickening of the ligamentum flavum and bone hyperplasia of the articular process, the space of the spinal canal is further reduced, which causes spinal stenosis to further aggravate waist and leg pain and even intermittent claudication [2]. Most scholars believe that a strict and rigorous systematic treatment plan from conservative treatment to final surgical treatment is required for $\mathrm{LDH}$ [3]. Patients with persistent pain after strict conservative treatment require surgical treatment [4-5].

Surgical treatments include traditional open surgery and new minimally invasive surgery. For general patients, open surgery has the disadvantages of large trauma, long postoperative recovery time and numerous complications. Besides, patients are often afraid of open surgery because of severe pain after surgery, which has forced clinicians to constantly explore new and safer minimally invasive surgery. Percutaneous endoscopic lumbar discectomy (PELD) is a rapidly developing new type of minimally invasive spinal surgery (MISS). Due to its advantages of a small incision, low blood loss, short hospital stay and exact curative effect, it has been 
affirmed and favored by a wide range of clinical surgeons in the treatment of LDH, spinal canal stenosis and even spinal fusion [6-8]. There are several surgical methods for percutaneous endoscopic technique, which are becoming more mature. However, each surgical method has its limitations. Regardless of the type of surgical approach of PELD, it is a single entrance (the observation and the operation ports are the same entrance).It is the same entrance in a tubular working sheath, and the co-axial relationship makes the visualization of the operation greatly restricted. Although the surgical field is microscopically enlarged, part of the field remains blocked, especially when a huge nucleus pulposus prolapses; the prolapsed nucleus pulposus is free to the posterior edge of the upper or lower vertebral body. Especially severe spinal canal stenosis and bilateral stenosis require decompression, it is difficult and challenging for surgeons [9-11] and unilateral biportal endoscopy (UBE) technology can reduce the difficulty of such operations to a certain extent.

UBE is a new minimally invasive surgery that combines open surgery and endoscopic surgery technologies. It utilizes arthroscopy and spine open surgery tools based on two separate small incisions to perform MISS. It is advantageous because of the large operating space, undisturbed tools and sightlines, small trauma, quick postoperative recovery, light postoperative pain, low postoperative infection risk and easier acceptance by patients [12-14]. Although UBE has gradually attracted the attention of most scholars, large-scale multi-center studies that strongly ratify the long-term efficacy of the operation are scarce. Additionally, comparative studies on UBE and Percutaneous endoscopic interlaminar discectomy (PEID) in the treatment of LDH are extremely rare. Herein, we explore and analyze the effectiveness and safety of UBE and PEID in the treatment of LDH with spinal stenosis to provide a reference for the application of UBE in the treatment of such diseases.

\section{Materials and methods}

\section{Ethics statement}


This retrospective study was approved by the Ethics Committee of the First People's Hospital of Yuhang District. Written informed consent was obtained from all patients.

\section{Subjects}

This study included 78 patients who received UBE or PEID at the First People's Hospital of Yuhang District, Hangzhou, China, from July 2018 to June 2020. After inclusion and exclusion criteria, 40 patients were included for analysis. All the 40 patients were operated on by the same surgeon (Dr. Gao). The inclusion criteria were as follows: (1) LDH with lumbar spinal canal stenosis was diagnosed and the pain was unresponsive after three months of rigorous conservative treatment and required surgical treatment. (2) The symptoms and signs were consistent with the imaging findings. (3) The liability segment is a single segment. (4) Treatment with UBE or PEID technology. The exclusion criteria were as follows: (1) the liability segment exceeds two segments. (2) History of previous posterior decompression surgery for diseased segments. (3) Significant lumbar instability and severe spondylolisthesis. (4) Patients with spinal infection, tumor, tuberculosis, etc. (5) Patients with coagulation disorders, mental diseases or other cognitive disorders that affect functional evaluation. (6) Missing imaging and follow-up treatment. (Fig.1)

\section{Surgical technique}

\section{Anesthesia methods and postures}

Epidural anesthesia was used in all patients. The patient was placed in the prone position on a radiolucent table to enable the use of a $\mathrm{C}$-arm fluoroscope. The patient was allowed to bend their hips and knees by adjusting the operating table to reduce the patient's anterior arch of the waist and open the intervertebral disc space as much as possible, which is beneficial in the access of the working channel. 


\section{PEID surgery}

First, in the body surface positioning surgery stage, the surgical segment was determined by positive C-arm fluoroscopy and a longitudinal incision of about 7.0 $\mathrm{mm}$ in length alongside the spinous process of $1.0-2.0 \mathrm{~cm}$ was made in the surgical segment to cut the deep fascia. A pencil-shaped dilation tube was placed at the junction of the superior lamina and the spinous process. The working channel was screwed along the expansion tube and the frontal and lateral perspective of the $\mathrm{C}$-arm machine was conducted again to determine its position. The dilation tube was removed and placed into the endoscope, which was continuously rinsed with normal saline. Under the microscope, fibrous adipose tissues on the surface of ligamentum flavum and vertebral plate were cleaned with nucleus pulposus forceps. After part of the upper and lower laminae bone mass was removed with a burr grinder and vertebral plate bone-biting forceps, the ligamentum flavum was gradually removed with gun forceps to expose the dural sac and nerve root. After the adhesion between the yellow ligament and the dural sac and the nerve root is separated, the working sheath tube is introduced into the spinal canal, and the dural sac and the nerve root are shielded by the baffle blade by rotating the sheath tube to avoid damage. The protruding nucleus pulposus tissue was gradually excised in different directions. The ablation electrodes were used for ablation, electrocoagulation and hemostasis. Meanwhile, the protruding intervertebral disc tissue was shrunk, and the intervertebral foramen was explored. If the stenosis was still persistent, the upper and the lower articular processes of the excised part were enlarged by the isthmus. If a patient had bilateral spinal canal stenosis, the working sheath was withdrawn from the spinal canal, part of the spinous process was removed, the working sheath was pushed into the opposite spinal canal from under the spinous process, the yellow ligament and prominent nucleus pulposus were removed and the intervertebral foramen was explored. Additionally, the patient was carefully examined for any sign of active bleeding after removing part of the upper and the lower articular processes. When the nerve root was loose and active and the dural sac was pulsatile, the patient withdrew 
from the endoscope and the working channel. The incision was then sutured.

\section{UBE surgery}

First, C-arm fluoroscopy from the anterior to the lateral position was used to determine the operating segment. A transverse incision was marked on the side with severe herniated disc stenosis, with the line of the pedicle inner edge as the center, 15 $\mathrm{mm}$ from the middle line of the responsible intervertebral space at the upper and lower distances. The cephalic incision (about $8 \mathrm{~mm}$ in length) was used as the observation channel and the caudal incision (about 12mm in length) was used as the working channel. A sharp knife was used to make a longitudinal incision of the skin and deep fascia, and a primary dilator was directly passed through the paravertebral muscle through the observation incision to reach the osseous surface of the vertebral plate. The soft tissues on the osseous surface of the vertebral plate were bluntly separated by a stepwise dilatation to form an observation channel. The endoscope sheath and the inner core were placed through the observation channel and the inner core was pulled out and placed into the arthroscope(Fig.2). The dilator was gradually inserted through the working incision to expand the soft tissue, form a working channel, open the perfusion system and continue the normal saline irrigation. The soft tissue structures obstructing the line of sight were cleaned and bleeding was stopped using a plasma radiofrequency knife to expose the bony tissue structure of the first field of view under the microscope, i.e., the junction of the spinous process and the lower edge of the upper vertebral body. This structure was also the initial target point for endoscopes and decompression devices. The operator operated with both hands and the left hand held the arthroscope. The arthroscope direction was adjusted to fully expose the surgical field. The instrument was operated with the right hand under the direct view of an endoscope. The bony laminae and the soft tissues on the surface of the ligamentum flavum were separated using a plasma radiofrequency knife and the inner edge of the articular process joint was exposed to the outside and the underside(Fig.3). The arthroscope was adjusted to expose the bony structure at the 
upper edge of the lower vertebral plate and complete the establishment of the extraspinal endoscopic workspace.

Part of the bone mass in the upper and the lower laminae of the target intervertebral space was removed using a power drill and gun forceps until the upper and the lower margins of the ligamentum flavum were free. The ipsilateral partial laminectomy and medial resection of the articular process were then performed using conventional open spine surgical instruments through the working channel(Fig.3). For severe osseous spinal canal stenosis or bilateral spinal canal stenosis, contralateral laminectomy and lateral recess resection and decompression were performed. The yellow ligament was kept intact during the whole decompression process to protect the nerves until the decompression was completed. Later, the adhesion between the dural sac and the ligamentum flavum was released and separated using a nerve stripper and the ligamentum flavum was separated to the caudal side to bite off the ligamentum flavum, thereby completing the establishment of the intraspinal working space(Fig.3). The dural sac was pushed open to expose the nerve root and the herniated intervertebral disc. The nerve root and the dural sac were pulled open by the assistant using the L-shaped nerve retractor through the operating channel. Bleeding was carefully stopped using a plasma radiofrequency knife to fully expose the intervertebral disc and remove the nucleus pulposus tissue(Fig.3). Observation under the microscope showed that the intraspinal dural sac had normal pulsation and external blood vessels were full, the nerve roots recovered to the normal shape and path, and there was no significant compression at the outlet(Fig.3). There was also no significant hemorrhage. After the instruments and the arthroscope were removed, residual lavage fluid was removed, the incision was sutured and the drainage tube was retained.

\section{Postoperative treatment and rehabilitation management}

All patients were treated with postoperative neurotrophic therapy and patients 
with postoperative acute pain were treated with non-steroidal anti-inflammatory drugs (NSAIDs). The patient was advised to get out of bed with waist circumference for 1 3 days after the operation. Strenuous exercise was not recommended, and bed rest was still advocated. Bed rest was still advocated. Additionally, the patient was required to avoid weight-bearing bending and twisting of the waist, as well as the moderate exercise of the lower back muscle.

\section{Outcome measures}

All patients underwent magnetic resonance imaging (MRI) and computed tomography (CT) examinations on the second postoperative day(Fig.1). Meanwhile, operation indexes such as operation time and a hospital stay of the patients in the two groups were recorded. The surgical bleeding volume could not be estimated accurately because all the patients underwent minimally invasive endoscopic therapy and received a whole-course lavage during the operation. The visual analog scale (VAS) score was used to assess the degree of low back pain in the patients. Scores at 1 day before operation and 1 day and 3 months after the operation and the last follow-up visit were recorded respectively. The Oswestry disability index (ODI) was used to assess the lumbar spine function. Scores were recorded preoperatively, at 1 day, and the last follow-up visit. The clinical effects of patients in the two groups were evaluated according to the Macnab standard.

\section{Statistical analysis}

SPSS 23.0 (SPSS Corporation, the US) statistical software was used for statistical analysis of the data and the measurement data were all expressed as $(\bar{X} \pm S)$. The VAS and ODI scores at different time points before and after the operation were compared using one-way repeated measures analysis of variance (ANOVA). The t-test was used for comparison between the two groups. A p-value of $<0.05$ was considered significant, and all tests were 2 sided. 


\section{Results}

\section{Patient demographic features}

In the UBE group, there were 20 patients (14 males and 6 females) aged $55.80 \pm$ 17.99 years old, including 7 and 13 cases at the L4/L5 and L5/S1 surgical segments, respectively. In the PEID group, there were 20 patients (15 males and 5 females) aged $39.89 \pm 12.81$ years old, including 8 and 12 cases at the L4/L5 and L5/S1 operation segments, respectively. There was no significant difference in basic information between the two groups. Demographic features of the patients are shown in Table 1.

Table 1 Demographic features of the patients between PEID and UBE groups

PEID UBE

\begin{tabular}{ccc}
\hline Case (n) & 20 & 20 \\
Male (n) & 15 & 14 \\
Female (n) & 5 & 6 \\
Age (year) & $39.89 \pm 12.81$ & $5.80 \pm 18.00$ \\
Hospitalization time (day) & $5.8 \pm 3.365$ & 7 \\
L4-5 (n) & 8 & 13 \\
L5-S1 (n) & 12 & \\
\end{tabular}

\section{Comparison of surgical indexes between two groups}

In the UBE group, the operation time and the hospital stay were $54.5 \pm 13.67$ minutes and $5.15 \pm 2.01$ days, respectively. In the PEID group, the operation time and the hospital stay were $64.8 \pm 15.51$ minutes and $5.8 \pm 3.37$ days, respectively. The operation time in the UBE group was better than that in the PEID group $(\mathrm{P}<0.05)$ (Fig.4), and the hospitalization days between the two groups had no significant 
difference $(\mathrm{P}>0.05)$.

\section{Comparison of VAS scores of back pain between the two groups}

The preoperative back pain scores in the UBE and the PEID groups were 5.75 \pm 0.91 and $5.35 \pm 0.99$, respectively but there was no significant difference between the two groups $(\mathrm{P}>0.05)$. The back pain scores of patients in the two groups after the operation were significantly improved as compared with those before the operation. The 1-day, 3-month and last follow-up scores in the UBE group were 3.35 $\pm 0.90,2.45 \pm 0.61$ and $1.25 \pm 0.55$, respectively, while scores in the PEID group were $3.3 \pm 0.57,2.45 \pm 0.51$ and $1.45 \pm 0.51$, respectively. The postoperative pain in both groups was significantly lower than that before surgery $(\mathrm{P}<0.05)$ but there was no significant difference between the two groups $(\mathrm{P}>0.05)$ (Fig.4).

\section{Comparison of VAS scores of leg pain between the two groups}

The preoperative leg pain scores in the UBE and the PEID groups were $7.15 \pm$ 0.99 and $7.15 \pm 0.99$, respectively, with no significant difference between the two groups $(\mathrm{P}>0.05)$. The leg pain scores of the two groups after the operation were also significantly improved as compared with those before the operation. The 1-day, 3-months and last follow-up scores in the UBE group were $3.15 \pm 0.59,2.3 \pm 0.47$ and $1.35 \pm 0.59$, respectively, while scores in the PEID group were $3.35 \pm 0.59,2.55 \pm$ 0.51 and $1.5 \pm 0.51$, respectively. Postoperative pain in both groups was significantly lower than that before surgery $(\mathrm{P}<0.05)$ but there was no significant difference between the two groups ( $\mathrm{P}>0.05)$ (Fig.4) .

\section{Comparison of ODI dysfunction index between the two groups}

The ODI score of the preoperative UBE group was $69.6 \pm 4.88$, while that of the PEID group was $69.6 \pm 4.88$ but there was no significant difference between the two groups $(\mathrm{P}>0.05)$. The scores in the UBE group at 3 months after surgery and the 
last follow-up were $24.2 \pm 2.75$ and $14.8 \pm 2.71$, respectively. The scores in the PEID group were $23.2 \pm 2.55$ and $14.65 \pm 2.64$, both of which were significantly improved as compared with those before surgery $(\mathrm{P}<0.05)$ but there was no significant difference between the two groups (P>0.05) (Fig.4).

\section{Clinical efficacy evaluation}

Among the 20 patients in the UBE group, 10 cases (50\%) were excellent, 6 cases (30\%) were good, 2 cases $(10 \%)$ were qualified and 2 cases $(10 \%)$ were poor. The excellent and good rate was $80 \%$. In the PEID group, 8 cases (40\%) were excellent, 6 cases $(30 \%)$ were good, 3 cases $(15 \%)$ were qualified and 2 cases $(10 \%)$ were poor. There was no significant difference between the two groups $(\mathrm{P}>0.05)$.

\section{Complications}

No noticeable intraoperative and postoperative complications were observed in the UBE group (including dural rupture, nerve root injury, cerebrospinal fluid leakage and infection). One patient in the PEID group experienced lower extremity transient pain three days after surgery and had no mobility. During hospitalization, the patient received intravenous dexamethasone, as well as oral treatment with NSAIDs and nutraceutical drugs. After three weeks, the symptoms disappeared. In addition, one patient developed numbness and discomfort of the lower limbs, had no obstacle in movement and had some weakness of foot dorsiflexion. After treatment with observation, functional exercise and anti-inflammation and analgesia, the symptoms resolved after three months.

\section{Discussion}

Percutaneous endoscopy is a commonly used minimally invasive surgical method in the treatment of $\mathrm{LDH}$. The main surgical approaches include the intervertebral foramen approach and the interbody approach. The development of the 
percutaneous intervertebral foramen approach benefited from the safe triangular working area in the intervertebral foramen first proposed by Kambin and colleagues [15] in 1972. Afterward, Yeung et al developed the intervertebral foramen endoscope system, YESS technology, via Kambin triangular approach in 1997, which enabled the endoscope to directly enter the intervertebral disc and cut off the nucleus pulposus tissue from the inside to the outside. However, it was impossible to completely cut off the nucleus pulposus tissue free from prolapse or prolapse and the indications for surgical treatment were relatively narrow. In 2003, Hoogland et al. [16] developed TESSYE endoscope technology based on YESS technology. The endoscope was inserted into the spinal canal through the intervertebral foramen zygapophyseal technology to operate under direct vision in a real sense [17]. However, the transforaminal technique exposed its shortcomings in the presence of a narrow foramen at the L5/S1 segment, hypertrophy of the transverse process of the lumbar 5 vertebral body and high iliac crest obstruction. Therefore, in 2006, Professor Ruetten [18] proposed the intervertebral approach based on the limitations of the transforaminal technique, making the endoscopic technology mature.

It is undeniable that percutaneous endoscopy has its unique advantages in MISS invasive spinal surgery, but some problems still remain exposed in daily surgery. First, the safe and effective puncture positioning technology enables most surgeons to learn a steep rise in the curve $[19,20]$. During the puncture, the puncture target should be positioned at the ventral of the articular process on the intervertebral foramen. In addition, inserting the puncture needle into the spinal canal should be avoided to prevent damage to nerves and dura mater. For less experienced surgeons, non-stop fluoroscopic positioning is required, which increases the risk of medical fluoroscopic exposure. Second, due to insufficient holding force of surgical instruments and partial soft tissue calcifications, such as intervertebral disc and/or ligamentum flavum calcifications, which cannot be completely removed, the residual tissues may cause persistent pain and numbness in the lower limbs [7]. Moreover, when performing a surgical operation under the observation of an endoscope (based on the diameter corresponding author: Zhichao Gao 
limitation of the tubular working sheath and the concentric and coaxial relationship between the surgical instrument and the working sheath and the endoscope), a surgeon may increase the operation difficulty and the risk of the nerve root and spinal canal injury because of the limitation of the visual field range of the working instrument during the surgical operation [10].

UBE technology is an emerging MISS technology. Based on two separate and independent small incisions, it utilizes arthroscopic tools to perform MISS with open spinal surgery tools. It has the advantages of small trauma, rapid postoperative recovery, mild postoperative pain, low risk of postoperative infection and easier acceptance by patients [12-14]. During operation, an operator operates the arthroscope with one hand and the working instruments with the other hand; hence, the operating instruments and the arthroscope have a v-shaped working mode. Compared with a single-door endoscope technology, the arthroscope and the working instruments are independent of each other and do not interfere with each other, thus the moving range of the instruments during the operation is wider and the limitation of observing and processing the operations on the contralateral side of the spinal canal and the intervertebral foramen area is reduced. UBE technology can not only use various surgical instruments of a single-door endoscope technology but also open surgical instruments, such as bone chisels and bone-biting forceps. Reducing the demand for surgical instruments can be more effective, safe and convenient in the treatment of laminae, ligaments and intervertebral discs, especially in the calcification of intervertebral discs and ligamentum flavum, making the technology more extensively in the treatment of diseases $[11,21,22]$. Compared with a single-door endoscope technology, the UBE technology learning curve is not so steep. First, for surgeons who are familiar with posterior spinal surgery and master endoscopic technology, under the observation of 30-degree arthroscopy, the surgical field of view is almost similar to that of open posterior surgery, making it easier for surgeons to find anatomical marks, thus ensuring the safety of the surgery [23]. Second, under the continuous perfusion of normal saline, the aqueous medium can not only clear the corresponding author: Zhichao Gao 
visual field of the operation compared with the air medium but also reduce the risk of postoperative infection $[13,14]$. Third, UBE has less demanding requirements on surgical body surface marking and the establishment of the observation and working channels through the puncture. It only needs to be placed at the pedicles of the upper and the lower vertebral bodies in the responsible intervertebral space. A relatively wide surgical field of view can be obtained by adjusting the angle of view of the arthroscope. In our previous study, the average hospital stay and the satisfaction rate of LDH patients with spinal stenosis treated by UBE technology were $5.15 \pm 2.01$ days and $80 \%$, respectively, which are similar to our current study [12]. However, the incidence of surgical complications in our patients was $0 \%$, which was superior to the current average incidence of surgical complications. The lack of surgical complications could be attributed to the small sample size of our previous study, which was also a major defect in the study. The average operation time in the present study was $54.5 \pm 13.67$ minutes, which was shorter than the 79.2 minutes in our previous study [12]. This may be due to our rich previous experience in posterior open spine surgery and advanced arthroscopic and percutaneous endoscopic techniques. Soliman et al. [24] required an average operation time of 93 minutes in the first 10 cases in the early stages of the UBE technology. With the development of technology and operation proficiency, the operation time in the subsequent 31 cases was significantly shortened to 60 minutes.

The UBE technology can be used not only in the treatment of LDH and spinal canal stenosis [14,25] but also in the treatment of lumbar spine fusion [26], distal syndrome [27] and cervical disc herniation [28]. Although its minimally invasive advantages are gradually accepted by most scholars, its disadvantages cannot be overlooked. The first is continuous normal saline perfusion. If there is no water flow and the drainage is not smooth, the vision of the operation can be blurred and the operator's identification of the tissue can be reduced, which might increase the nerve and dural sac damage during the operation. In the case of dural sac rupture, the operation ended prematurely. Moreover, continuous high-water pressure can cause corresponding author: Zhichao Gao Email: leadyourdream@163.com 
back muscle tissue edema. Second, although this technique can be applied in open surgery and could bring a series of conveniences, more blood oozing from the bone surface is inevitable. Although radio frequency technology can be utilized to stop bleeding and bone wax, gelatin sponge and brain cotton applied in compression hemostasis, hemostasis may not be completely achieved; the water medium mixed with blood is more turbid than the air medium, thus blurring the surgical field of view. Moreover, if there is more bleeding after surgery, the spinal hematoma is likely to be formed, hence the need to place an incision drainage tube after routine surgery.

Our insights into the UBE technology are focused on the following: (1) Arthroscopic radiofrequency can be used at the junction of the spinous process and vertebral plate to speed up the establishment of the operation space and save operation time. However, when used for hemostasis and soft tissue treatment in the spinal canal, we recommend replacement of the radiofrequency tissue under the endoscope or reduction of the radio frequency power to avoid nerve and dural sac damage.(2) For intraoperative water perfusion, a differential pressure at $40-50 \mathrm{~cm}$ height should be maintained (a pressure pump is not required for continuous perfusion) to reduce complications after surgery such as headache. Additionally, to maintain the patency of the water flow, a new water flow exclusion channel could be established outside the pedicle or a special half-tube drainage tube can be used. (3) Panoramic observation should be performed on the cephalic side using a 30-degree arthroscope to operate after clear tissue identification is ensured. When treating the ipsilateral intervertebral foramen, the arthroscope can be erected to vividly observe the condition of the ipsilateral intervertebral foramen and perform the treatment according to needs. For scholars who are not familiar with the 30-degree arthroscope, the 0-degree arthroscope can still be used but the scope of the operation field is smaller than that of the 30-degree arthroscope. 


\section{Conclusion}

Although our study has shown that the UBE technique can achieve the same surgical benefits as the percutaneous endoscopic technique and reduce the operation time and fluoroscopic exposure risk, there is currently a paucity of long-term follow-up and large-sample randomized controlled studies. Therefore, long-term follow-up studies with large samples should be conducted in the future to further ratify the reliability of the UBE technique.

\section{Statement}

Abbreviations

UBE: Unilateral biportal endoscopy

PEID: Percutaneous endoscopic interlaminar discectomy

LDH:Lumbar disc herniation

PELD: Percutaneous endoscopic lumbar discectomy

\section{Funding}

Not applicable.

\section{Acknowledgements}

Not applicable.

\section{Availability of data and materials}

The data and materials might be obtained from the corresponding author upon request.

\section{Authors' contributions}

B.Shen, X.Pan, and Z.Gao contributed to the conception and design of the study, performance of the experiments, data analysis and acquisition, and manuscript writing. D.Dai and M.Zhang designed the study and analyzed the data. X.Qian and D.Ruan contributed to the data acquisition. Y.Zhu edited the manuscript. The authors read and approved the final manuscript.

\section{Ethics approval and consent to participate}

This study was conducted at $t$ The First People's Hospital of Yuhang District, and permission was obtained from the hospital's ethics committee.

The authors had to obtain patient consent before enrolling participants in this study. 


\section{Competing interests}

The authors declare that they have no competing interests.

\section{References}

[1] Zhou Muwang, Yue Shouwei, HE Chengqi, et al. Rehabilitation treatment of lumbar disc herniation Consensus of Chinese Experts[J]. Chinese Journal of Rehabilitation Medicine, 2017, 32(2):129-135.

[2]Tomkins-Lane C, Melloh M, Lurie J, et al. ISSLS prize winner: consensus on the clinical diagnosis of lumbar spinal stenosis: results of an international Delphi study. Spine. 2016;41(15):1239-46.

[3] Patwardhan R V, Hadley $M$ N . History of surgery for ruptured disk[J]. Neurosurgery Clinics of North America, 2001, 12(1):173.

[4] Kin TW ,Oh CH ,Shim YS et al. Psychopathological influence of lumbar disc herniation in male adolescent[J]. Yonsei Medical Journal, 2013, 54(4):135-140

[5] González M E, Garcia-Cosamalon J, Cosamalon-Gan I, et al. Biology and mechanobiology of the intervertebral disc[J]. Neurocirugia (Asturias, Spain), 2017, 28(3): 135.

[6]Ahn Y. Transforaminal percutaneous endoscopic lumbar discectomy: technical tips to prevent complications[J]. Expert review of medical devices, 2012, 9(4): 361-366.

[7]Ahn Y, Lee S H, Park W M, et al. Percutaneous endoscopic lumbar discectomy for recurrent disc herniation: surgical technique, outcome, and prognostic factors of 43 consecutive cases[J]. Spine, 2004, 29(16): E326-E332.

[8]Mobbs R J, Phan K, Malham G, et al. Lumbar interbody fusion: techniques, indications and comparison of interbody fusion options including PLIF, TLIF, MI-TLIF, OLIF/ATP, LLIF and ALIF[J]. Journal of spine surgery, 2015, 1(1): 2.

[9]Choi I, Ahn J O, So W S, et al. Exiting root injury in transforaminal endoscopic discectomy: preoperative image considerations for safety[J]. European Spine Journal, 2013, 22(11): 2481-2487.

[10] Nowitzke A M. Assessment of the learning curve for lumbar microendoscopic discectomy[J]. Neurosurgery, 2005, 56(4): 755-762.

[11]Kim S K, Kang S S, Hong Y H, et al. Clinical comparison of unilateral biportal endoscopic technique versus open microdiscectomy for single-level lumbar discectomy: a multicenter, retrospective analysis[J]. Journal of orthopaedic surgery and research, 2018, 13(1): 22.

[12]Lin G X, Huang P, Kotheeranurak V, et al. A systematic review of unilateral Biportal endoscopic spinal surgery: preliminary clinical results and complications[J]. World neurosurgery, 2019, 125: 425-432.

[13]Heo D H, Quillo-Olvera J, Park C K. Can percutaneous biportal endoscopic surgery achieve enough canal decompression for degenerative lumbar stenosis? Prospective case-control study[J]. World Neurosurgery, 2018, 120: e684-e689.

[14]Min W K, Kim J E, Choi D J, et al. Clinical and radiological outcomes between biportal endoscopic decompression and microscopic decompression in lumbar spinal stenosis[J]. Journal of Orthopaedic Science, 2020, 25(3): 371-378. 
[15] Kambin P, Gellman H. Percutaneous lateral discectomy of the lumbar spine a preliminary report[J]. Clinical Orthopaedics and Related Research ${ }^{\circledR}, 1983,174$ : 127-132.

[16] Hoogland T. Transforaminal endoscopic discectomy with foraminoplasty for lumbar disc herniation[J]. Surg Tech Orthop Traumatol, 2003, 40(40): 55-120.

[17] Hoogland T, Schubert M, Miklitz B, et al. Transforaminal posterolateral endoscopic discectomy with or without the combination of a low-dose chymopapain: a prospective randomized study in 280 consecutive cases[J]. Spine, 2006, 31(24): E890-E897.

[18] Ruetten S, Komp M, Godolias G. A new full-endoscopic technique for the interlaminar operation of lumbar disc herniations using 6-mm endoscopes: prospective 2-year results of 331 patients[J]. min-Minimally Invasive Neurosurgery, 2006, 49(02): 80-87.

[19] Lee S H, Kang B U, Ahn Y, et al. Operative failure of percutaneous endoscopic lumbar discectomy: a radiologic analysis of 55 cases[J]. Spine, 2006, 31(10): E285-E290.

[20] Yeung A T, Tsou P M. Posterolateral endoscopic excision for lumbar disc herniation: surgical technique, outcome, and complications in 307 consecutive cases[J]. Spine, 2002, 27(7): 722-731.

[21]Heo D H, Son S K, Eum J H, et al. Fully endoscopic lumbar interbody fusion using a percutaneous unilateral biportal endoscopic technique: technical note and preliminary clinical results[J]. Neurosurgical Focus, 2017, 43(2): E8.

[22]Kim J E, Choi D J. Bi-portal Arthroscopic Spinal Surgery (BASS) with $30^{\circ}$ arthroscopy for far lateral approach of L5-S1-Technical note[J]. Journal of orthopaedics, 2018, 15(2): 354-358.

[23] Kang S S, Lee S C, Kim S K. A novel percutaneous Biportal endoscopic technique for symptomatic spinal epidural Lipomatosis: technical note and case presentations[J]. World neurosurgery, 2019, 129: 49-54.

[24]Soliman H M. Irrigation endoscopic discectomy: a novel percutaneous approach for lumbar disc prolapse[J]. European Spine Journal, 2013, 22(5): 1037-1044.

[25] Eum J H, Heo D H, Son S K, et al. Percutaneous biportal endoscopic decompression for lumbar spinal stenosis: a technical note and preliminary clinical results[J]. Journal of Neurosurgery: Spine, 2016, 24(4): 602-607.

[26]Park M K, Park S A, Son S K, et al. Clinical and radiological outcomes of unilateral biportal endoscopic lumbar interbody fusion (ULIF) compared with conventional posterior lumbar interbody fusion (PLIF): 1-year follow-up[J]. Neurosurgical review, 2019, 42(3): 753-761.

[27] Heo D H, Sharma S, Park C K. Endoscopic Treatment of Extraforaminal Entrapment of L5 Nerve Root (Far Out Syndrome) by Unilateral Biportal Endoscopic Approach: Technical Report and Preliminary Clinical Results[J]. Neurospine, 2019, 16(1): 130.

[28] Park J H, Jun S G, Jung J T, et al. Posterior percutaneous endoscopic cervical foraminotomy and diskectomy with unilateral biportal endoscopy[J]. Orthopedics, 2017, 40(5): e779-e783. 


\section{Figures}

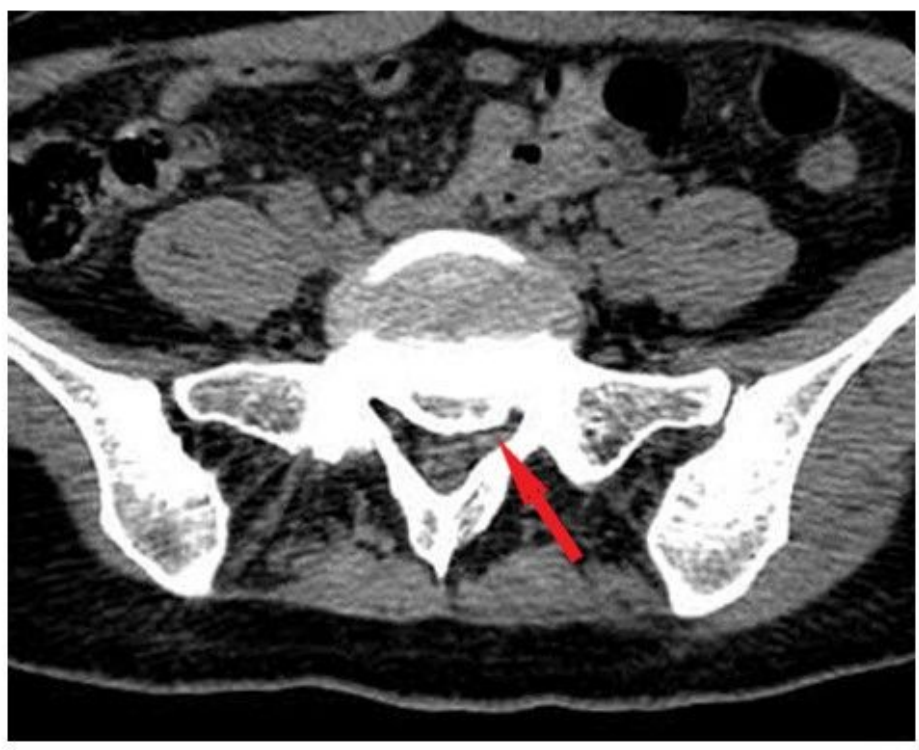

(a)

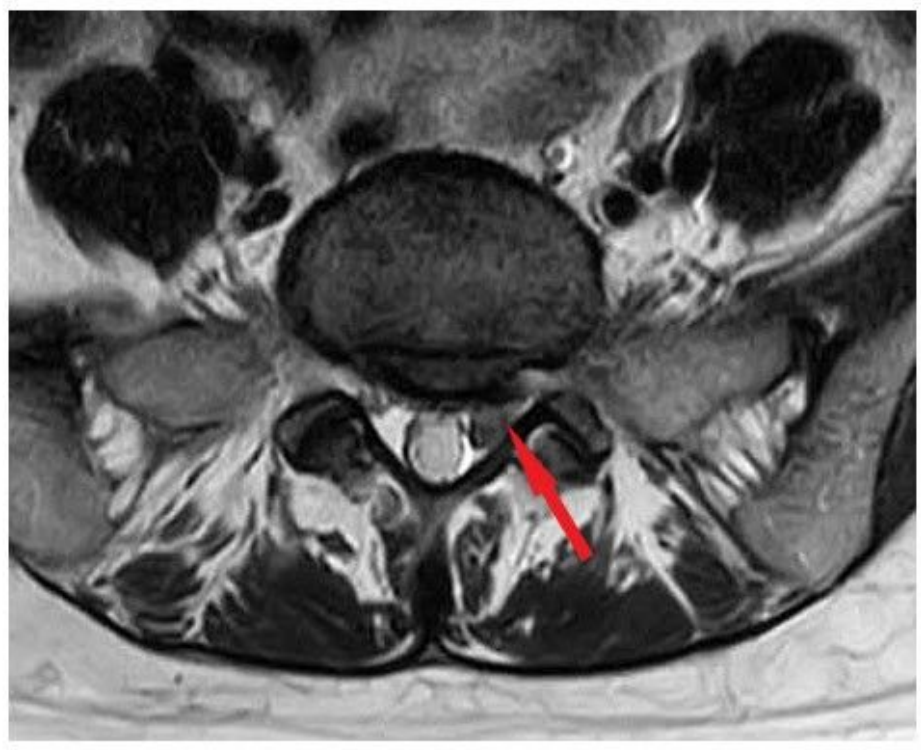

(c)

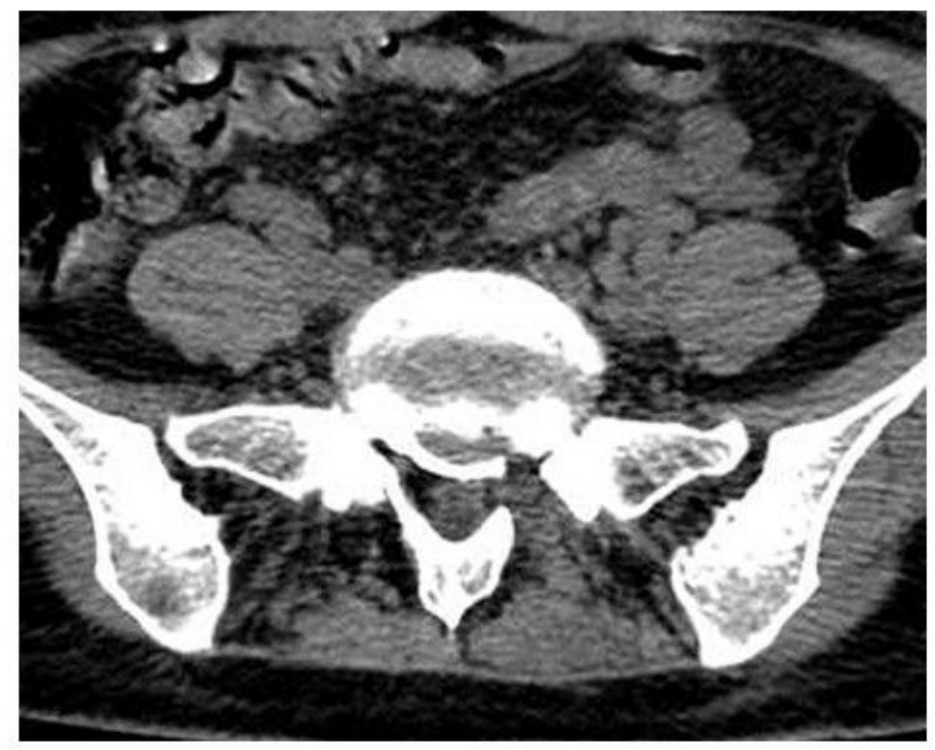

(b)

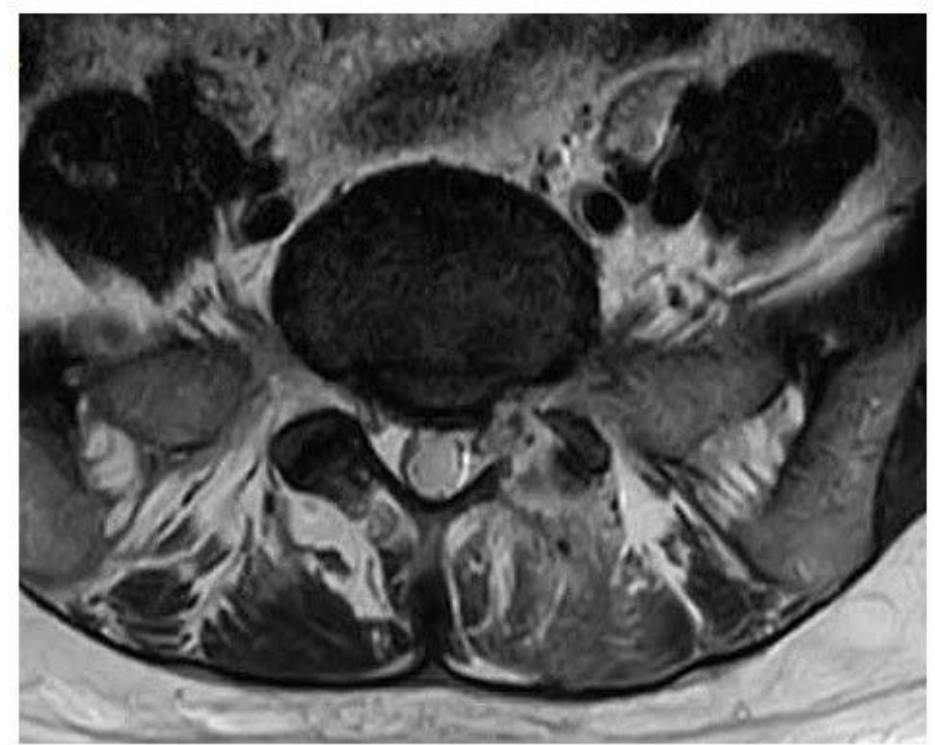

(d)

\section{Figure 1}

The preoperarive and postoperative images of a case. a, c Preoperative CT (a) and MRI (b) images showing herniated nucleus pulposus with calcification at the L5-S1 level (arrows). b, d Postoperative CT (b) and MRI (d) images showing adequate decompression of the neural structures. 


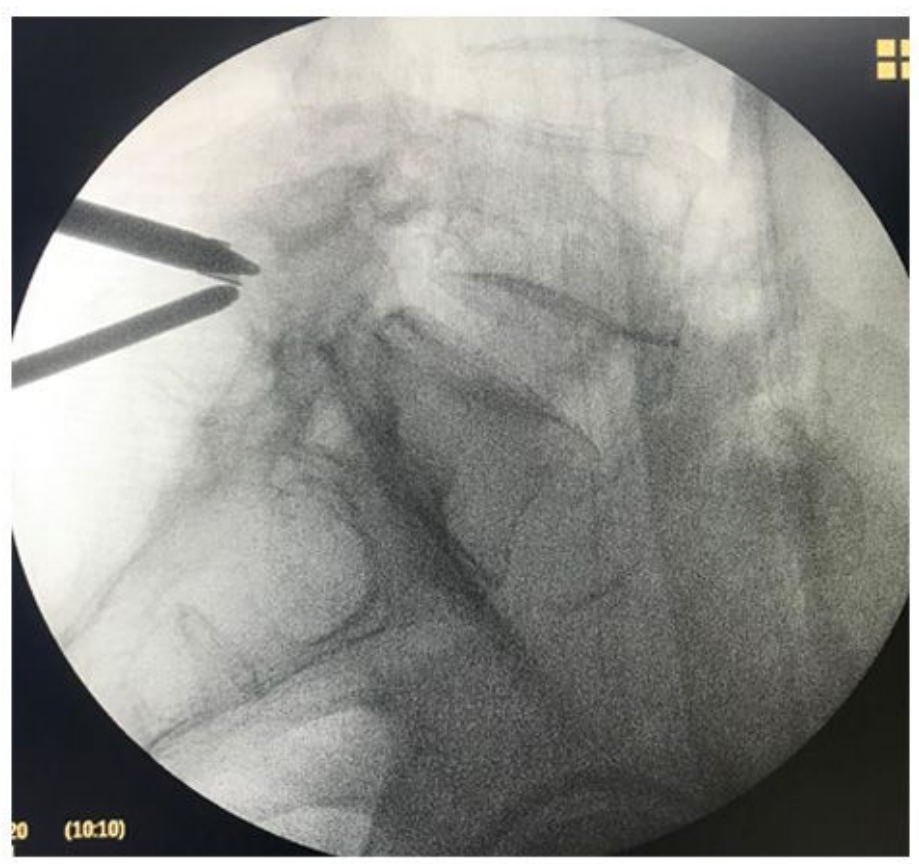

(a)

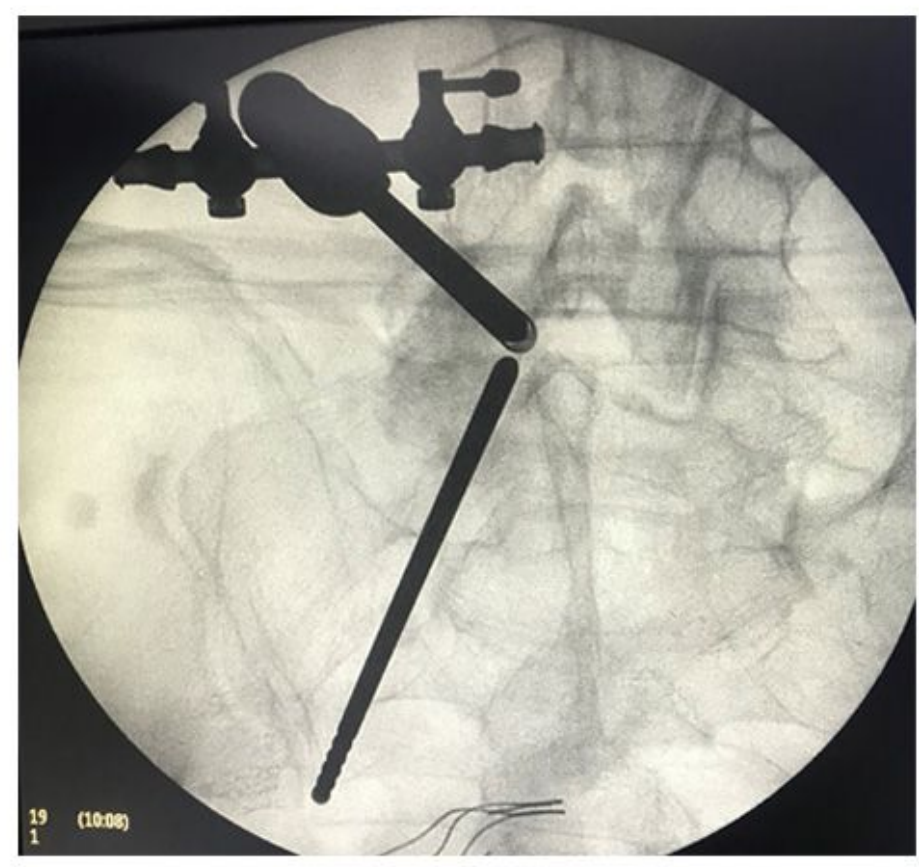

(b)

\section{Figure 2}

The localization of the working cannula. a, b Radiography showing the working cannula has successfully reached the operation area at the L5-S1 levels. 


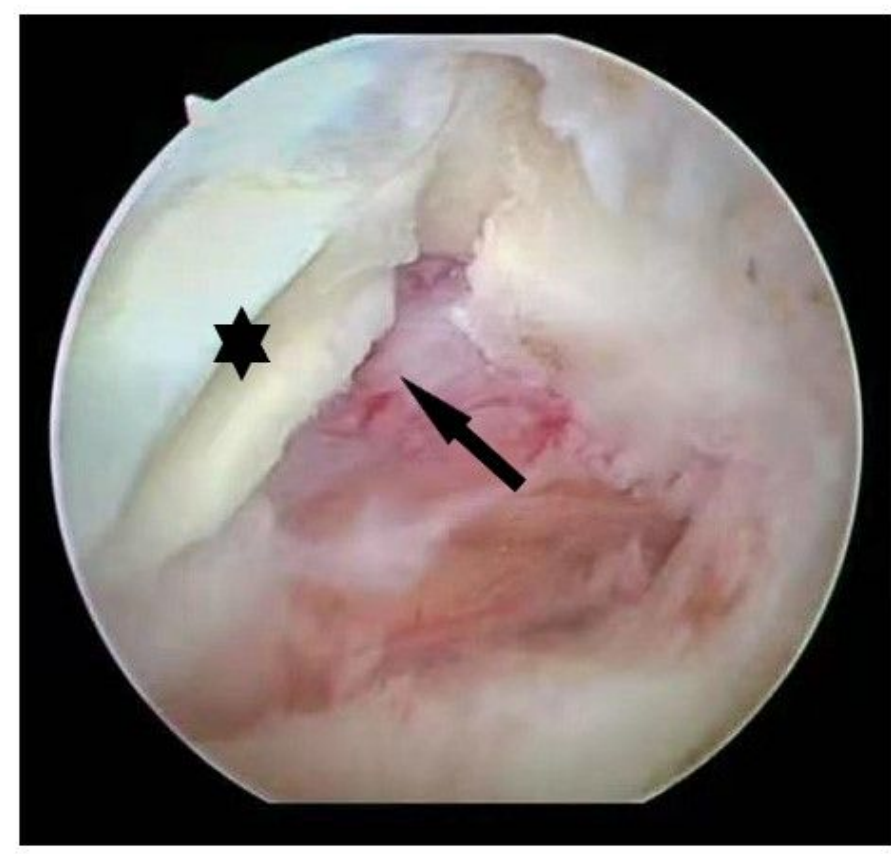

(a)

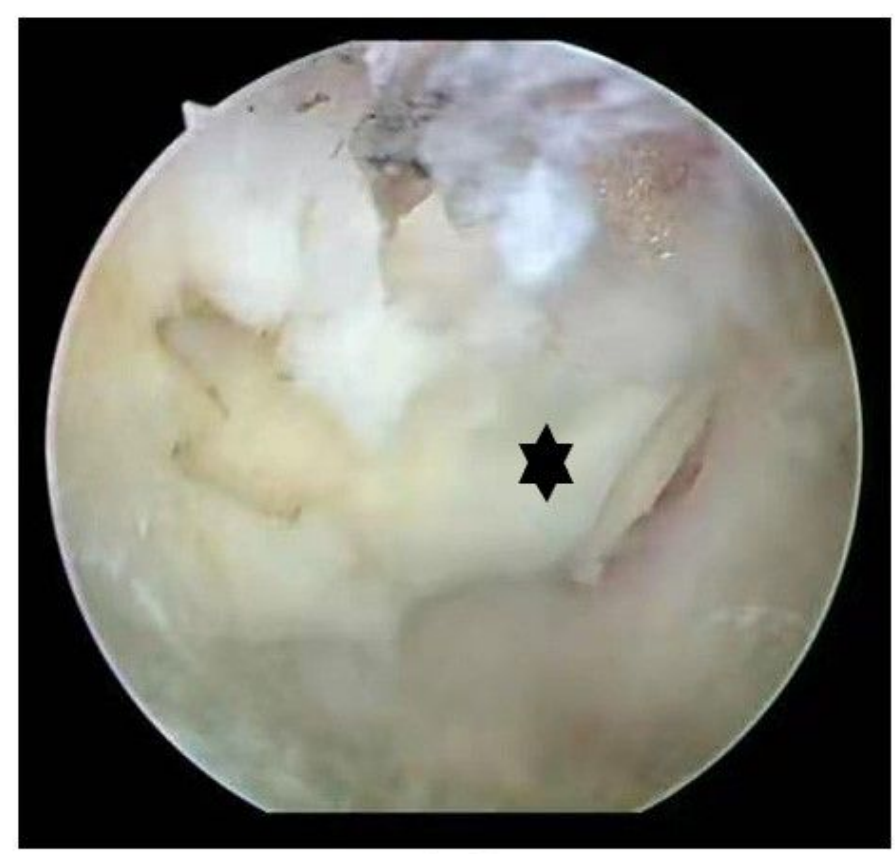

(c)

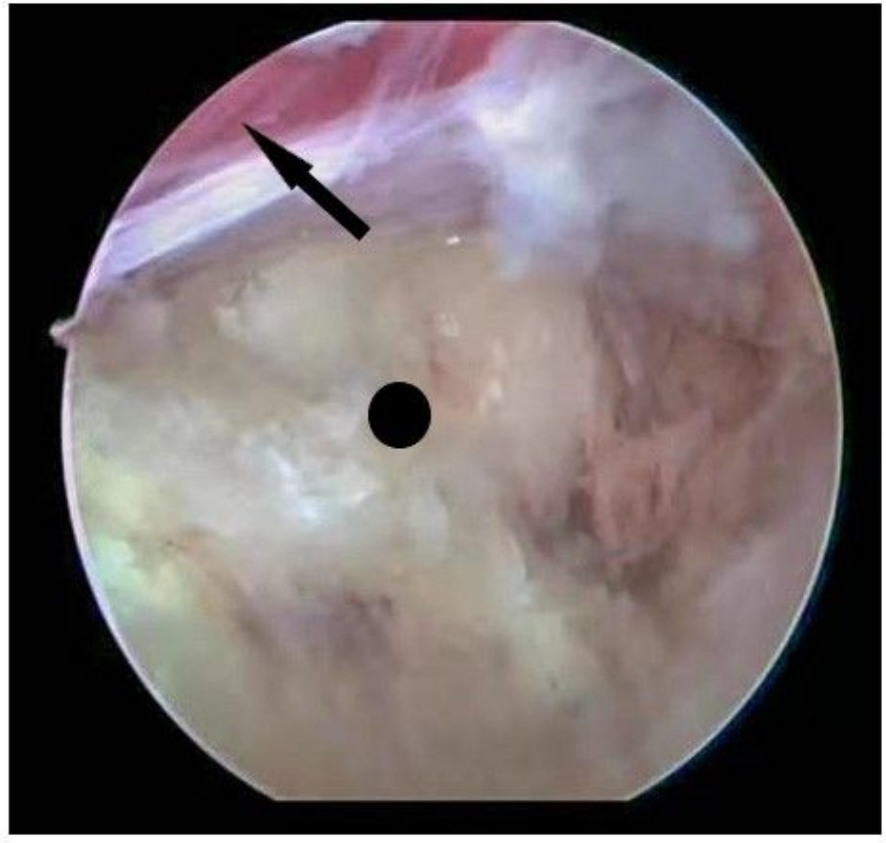

(b)

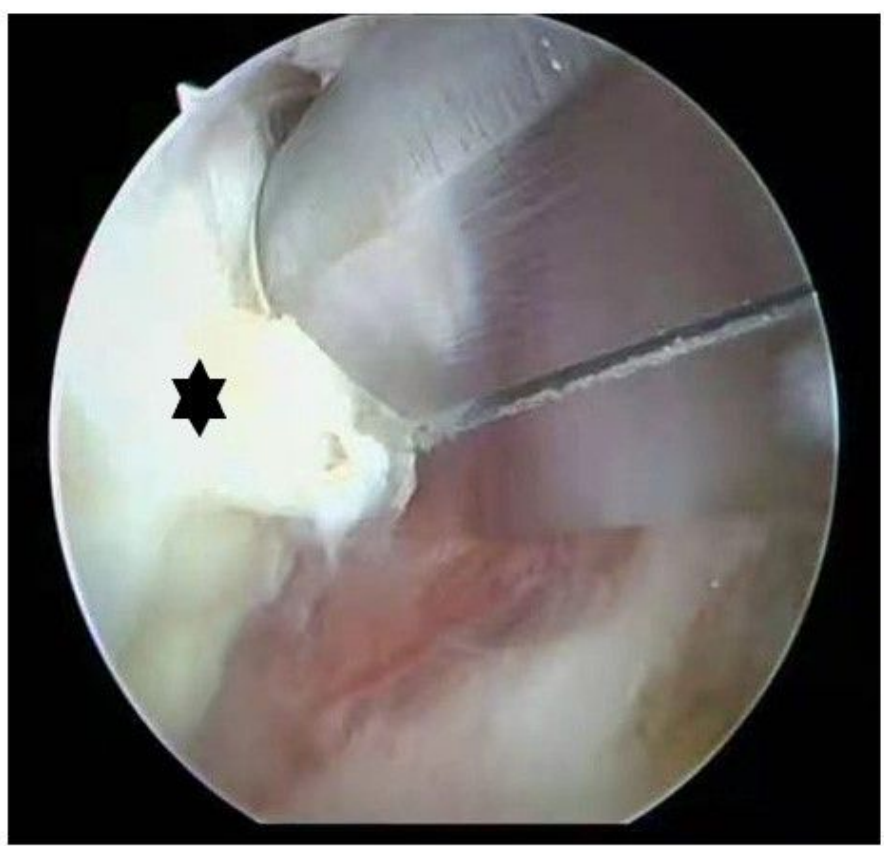

(d)

\section{Figure 3}

Endoscopic views of the surgical procedure. (a) The lumbar vertebral canal was opened, and the nerve root was exposed. (b) The symptomatic disc was removed, and then the calcified tissues was removed. (c) A partial laminectomy was performed and the ligamentum flavum was exposed. (d) Soft tissue, including the ligamentum flavum, was removed with a rongeur and nucleus pulposus forceps 
Surgery Time

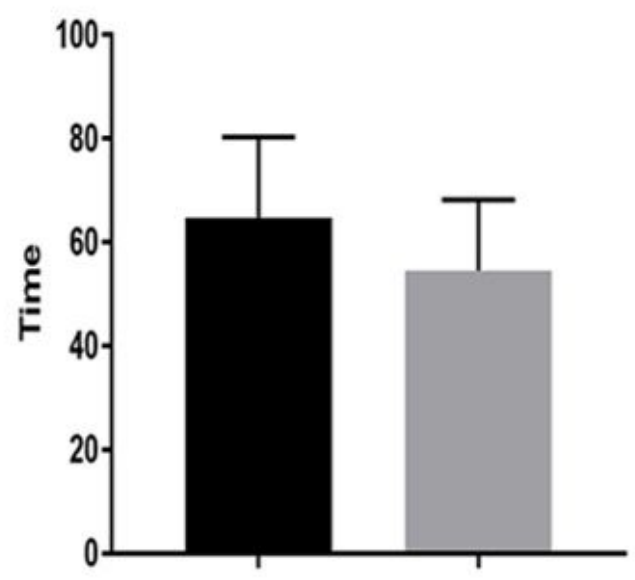

(a)

VAS of Back

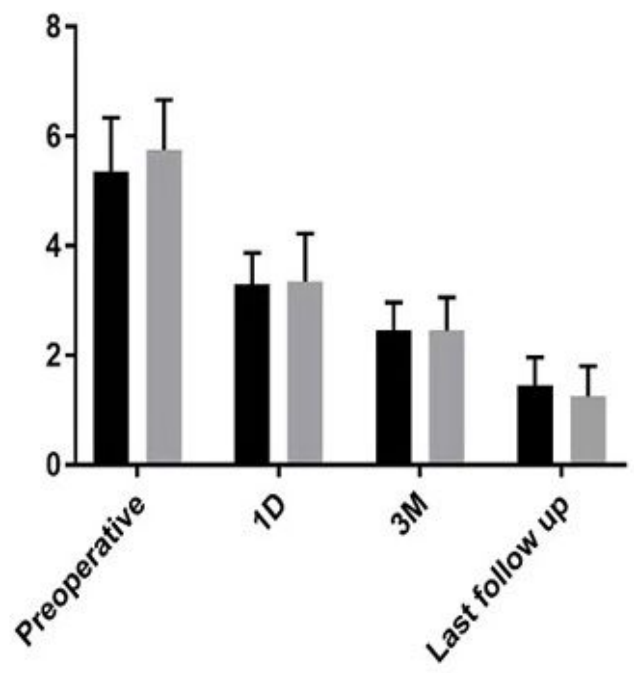

(c)
ODI

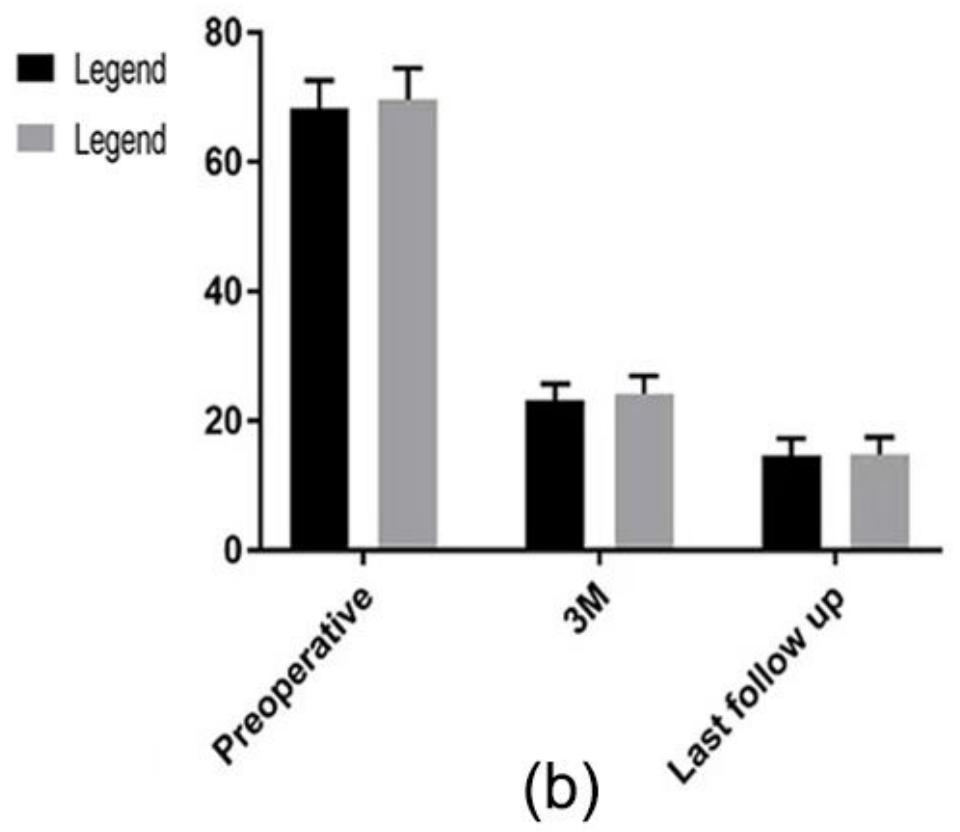

VAS of leg

- PEID
- UBE

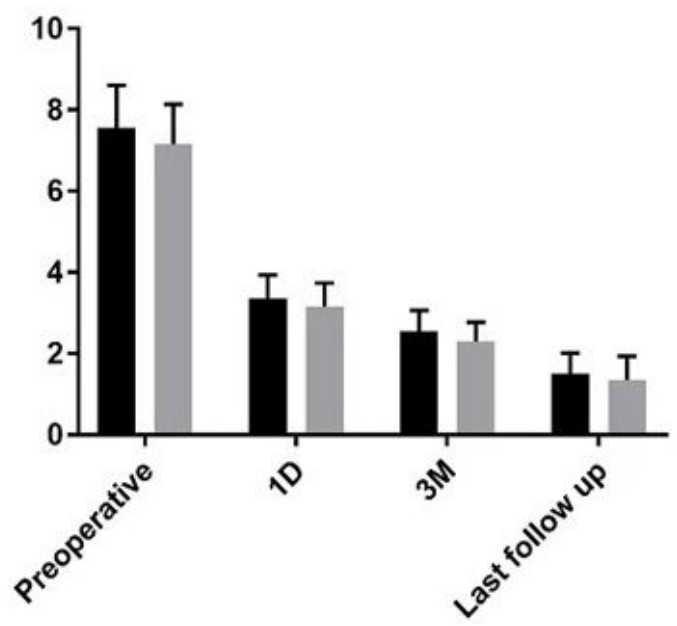

(d)

Figure 4

Various clinical scores. (a) Primary to compare the surgery time between the two groups. (b) Lumbar ODI score of two groups before the operation and at different time points postoperation. (c) Back pain VAS score of two groups before the operation and at different time points postoperation. (d) Leg pain VAS score of two groups before the operation and at different time points postoperation. (Note: To compare with the control group, The UBE has advantages in surgery time, $\mathrm{P}<0.05$ ) 